\title{
Modified Math Client-Server Application for E-Learning
}

\author{
M. Yakubu \\ Department of Computer \\ Science, Adamawa State \\ University, Mubi
}

\author{
C. U. Ngene \\ Department of Computer \\ Engineering, University of \\ Maiduguri
}

\author{
Y. Gambo \\ Department of Computer \\ Science, Adamawa State \\ University, Mubi
}

\begin{abstract}
Technology plays a vital role in global academic community. However, majority of learners still have extreme mathematical timidity. The demand to make learning process of mathematics simplified is increasing. As the modern life activities are getting completely centered on the Internet, providing an infrastructure for bringing mathematical computation over the Internet will greatly promote the mathematics learning. This dissertation makes two contributions. First, development of a modified math clientserver application for easy mathematical computation on any network. Secondly, the work presents concepts involved in creating client server network applications using the TCP/IP socket programming, this work was realized using java technology. The application runs on both intranet and internet, allowing clients to interact and access mathematical functions provided by the server using request - reply protocol.
\end{abstract}

\section{General Terms}

Math-client-server, mathematical functions and TCP/IP.

\section{Keywords}

e-learning, math-client-server, client-server communication, mathematical functions.

\section{INTRODUCTION}

The need to make learning process of mathematics simplified is increasing. This is because mathematics is important for every learner and society as the foundation for other subjects. Unfortunately, several learners see mathematics as a difficult subject, as a result, when they hear the term mathematics, they have this special fear. The majority of learners have extreme mathematical timidity. The issue of fear and hatred for mathematics is mainly due to environmental or personal reasons [1].

Communication between two or more computers takes place due to a network. A "Computer Network" is a collection of autonomous computers interconnected by a single technology. Connections can be made with the help of hardware like copper wires, fiber optic cables or waveforms like Bluetooth, infrared, Wi-Fi and satellites [2].

Different technologies such as xml, xsl and both MathML and Open- Math made it possible to bring mathematics to the internet [3].

A Client-Server system has one or more client processes and one or more server processes, and a client process can send a query i.e. (requests services) to any one of the server process. Clients are responsible for user-interface issues and regarded as Front-end Application. Servers manage data and execute transactions in other word provides services to the clients; they are said to be Back-end Application. Thus, a client process could run on a personal computer and send queries to a server running on a mainframe. [4].

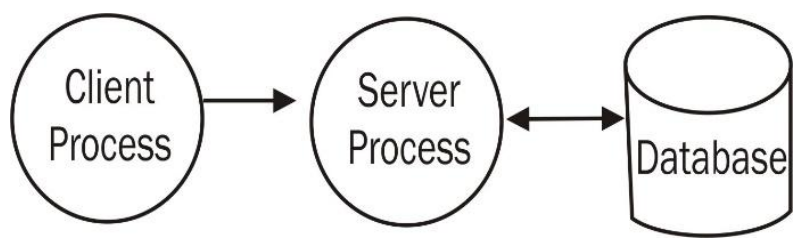

Figure 1: Client Server Architecture

According to [5] the distribution of services in client/server increases the susceptibility of these systems to damage from viruses, fraud, physical damage and misuse than in any centralized computer system. With businesses moving towards multi-vendor systems, often chosen on the basis of cost alone, the security issues multiply. That, security has to encompass the host system, PCs, LANs, workstations, global WANs and as well as the users.

E-learning refers to the use of information and communication technologies to enable the access to online learning/teaching resources [6].

The learning process of mathematics, with its abstract ideas and concepts, can be simplified by creating online models. This is to take advantage of opportunities presented by the internet, by providing an infrastructure for bringing mathematical computation over all networks. Different technologies such as xml, xsl and both MathML and OpenMath made it possible to bring mathematics to the internet [3].

This work presents a clear need for learning mathematics; by presenting how technology can improve math's learning process based on electronic models as compared to the existing method. The development of Modified Math ClientServer application had made mathematical computation easy. This is to make mathematics learning more effective by challenging learners to become more active participants, more confident and effective learners [7].

\section{LITERATURE REVIEW}

Simple Math server in particular has been implemented using different technologies and models but has some bottlenecks [8]. The existing simple math server frameworks can perform basic math operations, not fault tolerant; has a greater computation overhead to ensure reliable delivery of packets, requires a 3-way handshake, the architecture is Internet-based, less mathematical functions deployed.

In the implementation of simple math-server one has to consider the specific protocol defined by the math-server and client communication. The program uses a very simple protocol operator: first value: second value. It is the math server's responsibility to understand this protocol and delegate to the proper operations such as add, sub, mul, or div. 


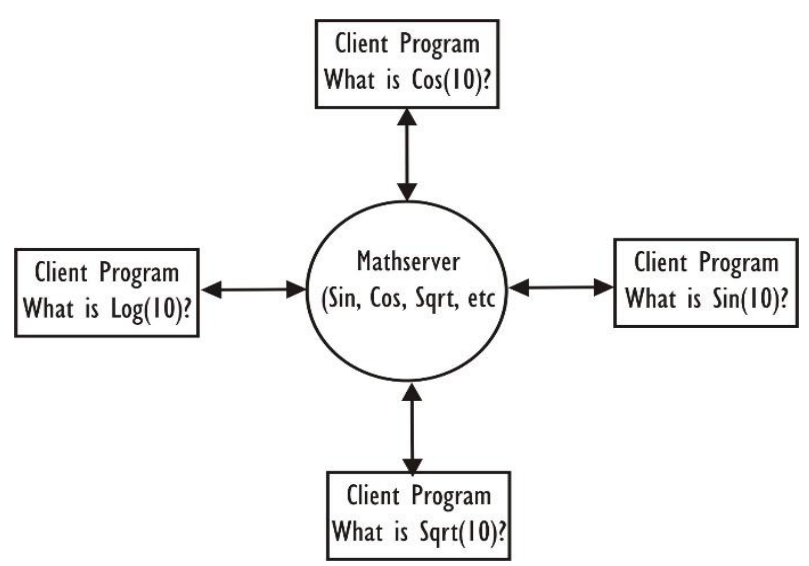

Figure 2: A socket-based math-server and clients, using the Master Worker Thread Model [9].

This research is focused on implementing modified math client-server application, using the Master Worker Thread Model. The work has optimized the existing simple math server by integrating more mathematical functions and developing a modified math client-server.

The modified math client-server run large computations at much faster than existing ones [10].

\subsection{Modified Math Client-Server Application}

Modified Math Client-Server application is easy to use has user-friendly GUI, it requires no prior networking skills it could be deployed in schools and any other educational learning centres. It occupies minimal computer memory also it is easy to setup. It makes use of simple client-server architecture. One file is installed on the "server", which would be the administrator's computer and the other file is installed on every machine in the network. On installing the server-side application, a list of all the active computers on the network is displayed. This then allows the server-side application to communicate with the client-side application.

The application has been developed using Java technology (Java Eclipse Platform) as its inbuilt libraries and objectoriented nature were very conducive to completing this work in the most effective and efficient way possible.

All the math functions that are in the server-side application are accessible from client application which enables the client not to have much task of processes to carry out. The client can make a specific request from math server and a respond can be made. Also, the client can communicate with the server online through chatting.

All the information collected by the network address translation (NAT) is stored in a database which is managed using MySQL. This allows the admin to review computer performance, efficiency, energy usage, data transfers and log reports of viruses, crashes and other data which help in determining the overall effectiveness of the software and functioning of the network in the organization.

\section{SYSTEM ARCHITECTURE}

\subsection{Working of the System}

The system relies on 2 components: The Server (Administrator's computer) and the Client (computers to be monitored).

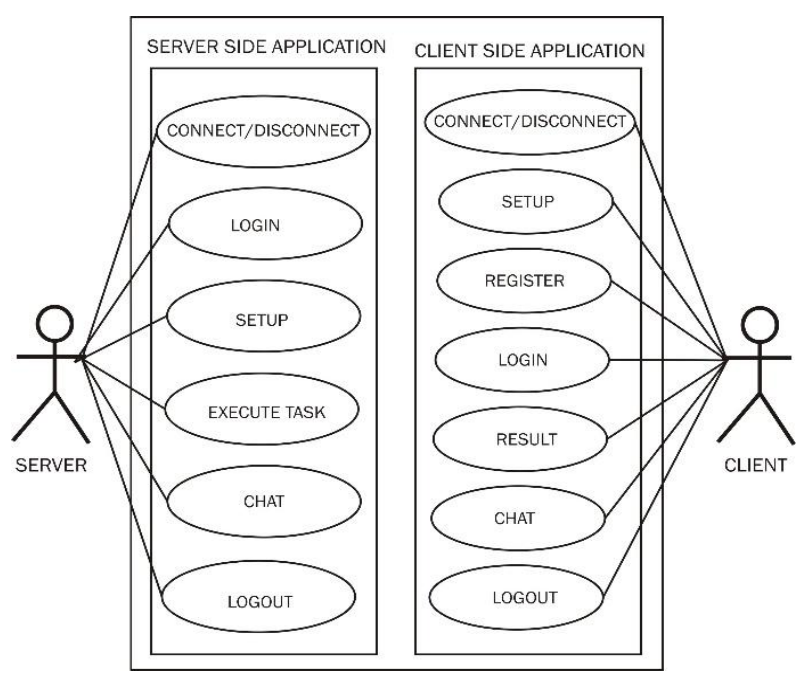

Figure 3: System Architecture (Use Case Diagram)

Client-side Functionality:

- Connect: for connecting the clients to the server. When the indicator displays red colour it indicates that the client is not connected to the server. When the indicator displays green colour it indicates that there is an establishment of connection between the client and the server.

- Disconnect: the disconnect button disconnect the client from the remote server computer which cannot allow any services from the server to be access by the client computer.

- Setup: For setting the necessary connection information such as PC Number, Remote Port Number and either of Host IP Address or Remote Server Host Name.

- Register: It enables the user to register himself so as to enable him use and utilize the available resources that is on the server.

- Login: The login button pops up the login window which require the users to login his details.

- Result: After the processes, the server sends the result of the processes to the client

- Chat: for enabling the user (clients) to chat with the administrator (Server) whenever the client wants more clarification on any of the math function found on the server.

- Logout: logout out the client from the server

Server -side Functionality:

- Connect/disconnect: for connecting the clients from the server. When the indicator displays red colour it indicates is not ready for connection. When the indicator displays yellow colour it indicates that the server is ready for connection with the client. When the indicator displays green colour it indicates that there is an establishment of connection between the server and the client.

- Setup: For configuring a server setting which enable the client to use the parameter for their connection.

- Chat: this pops up the chat panel, which displays the list of logged in users with whom you can chat with 


\subsection{DATA FLOW DIAGRAM}

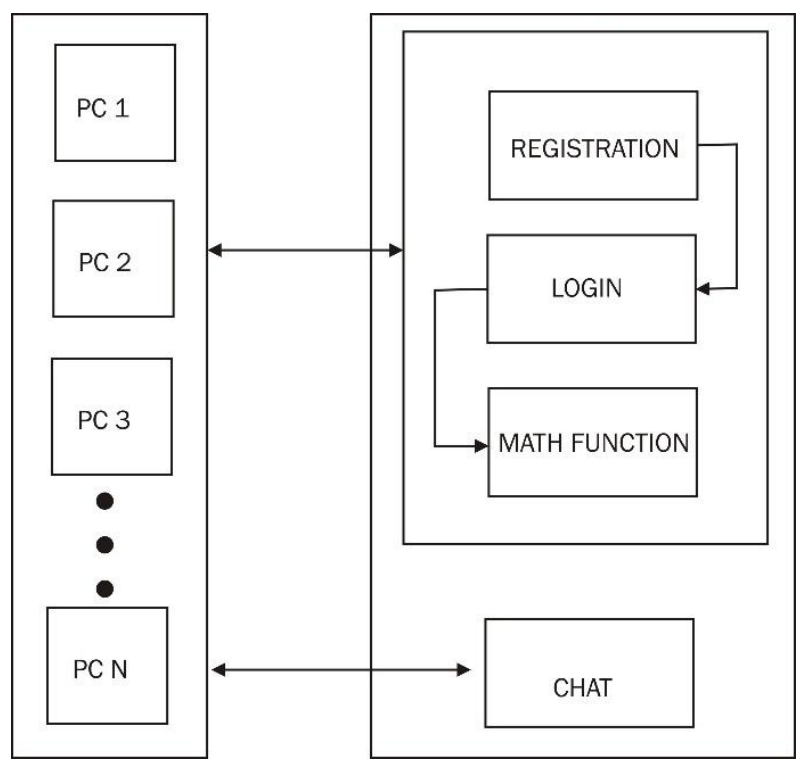

Figure 4: Data flow - Client request from server and server respond to the client

\subsection{Sequence of Events}

The sequences of events of the Modified Math Client Server Application are shown below:

Server Side:

1. Server computer and the application Startup.

2. Server initiate the network settings for client to connect: such as PC Number, Remote Port Number and either of Host IP Address or Remote Server Host Name.

3. Ready for requests from the client

Client Side:

1. Client computer and the application Startup.

2. Setup a network setting for the client computer for connection, information requires are PC Number, Remote Port Number and either of Host IP Address or Remote Server Host Name.

3. Client connect to the server and ready for communication with the server which indicates the connection button as green.

4. Client Register himself to the server for resources utilization.

5. Login to the server for onward request (if validate right then get access if no then abort).

6. Client send request for any of the available math function on the server for a result.

7. Client received a response of its request from the server

8. Initiates a chat session with the server.

\section{METHODOLOGY}

For proper implementation of this application, it is to be implemented in a networked environment using either a wired or a wireless medium, the client side of the application was installed on the client computer, while the server side of the application was installed on the server. The client's computer was setup to the network parameter that the server was presetup and tested with 30 desktop computers and 15 laptops computers which runs concurrently accessing the server requesting different mathematical functions services and at the same time the server sends the results to the client's computers that made the request.

Although the specification of the computer varies, the time taken to access and also get the result from the server varies depending on the capacity of the computer systems. The operating system used also varies though it does not affect the performance of the application.

The server side:

1. Installed server-side application

2. Preconfigured the network for client to be connected

3. Chat with the client

Then the following operations were performed at the client side after installation of the application:

1. Installed the client-side programs

2. Setup the network parameter for the server connection

3. Connect the server computer to the client, ran a system

4. Registered a user for server accessibility

5. Login and accessed the available resources on the server (the math functions and chatting capability).

\subsection{Requirements}

For the software to run correctly and efficiently it require a computer with the following specification:

Hardware Requirements

Processor: $\quad$ Pentium IV $2 \mathrm{GHz}$ and Above

RAM :2GB RAM and above

Hard disk: $\quad$ 80GB or more

Monitor : $\quad 15$ " CRT or LCD monitor

Keyboard: $\quad$ Normal or Multimedia

Mouse: Compatible mouse

Software Requirements

Developing Tool: java (JVM: JDK 7)

Operating System: Windows XP with service pack 3 or later versions of windows

A typical LAN networks.

\section{SYSTEM TESTING}

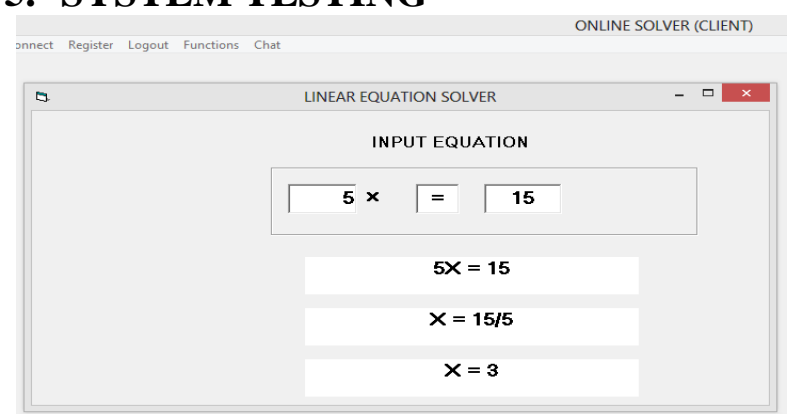

Figure 5: Linear equation

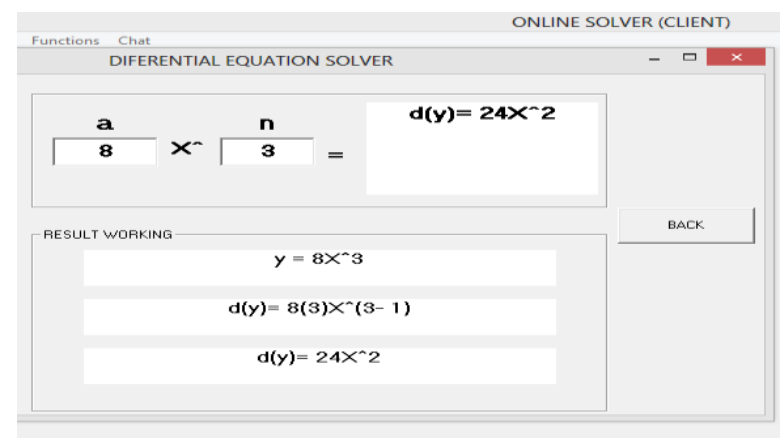

Figure 6: Differential Equation 


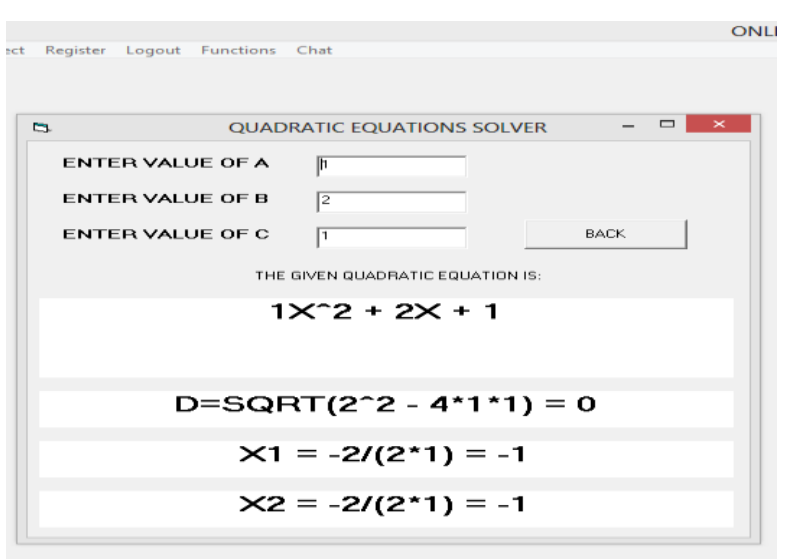

Figure 7: Quadratic Equation

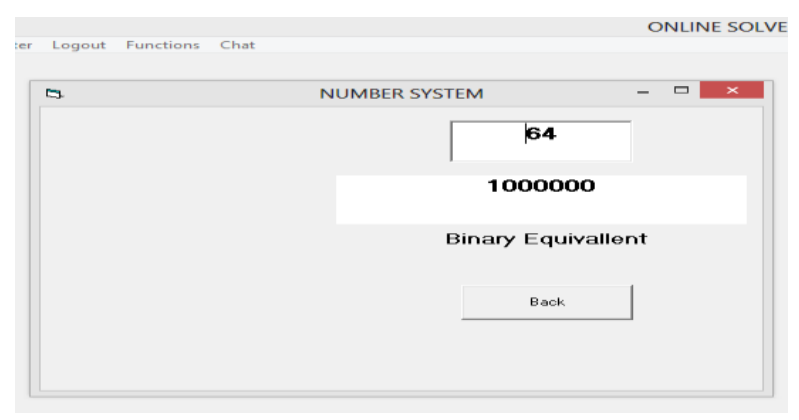

Figure 8: Number System

\section{CONCLUSION}

This paper discussed the development of math client-server application for easy mathematical computation on any network for the multiple clients communicating through a server in a TCP/IP network. This was achieved using masterworker thread model, the approach allows clients to access mathematical computational facilities through a uniform set of network protocols. A real time interactive session for client/server system solution has been developed to help mathematics learners. The system was designed and run on the Java-eclipse and MySQL as its database.

\section{FUTURE WORK}

i. Development of mathematical notation that can be use during chatting

ii. Incorporating more mathematical functions

iii. Development of a printing window, where a client can print the solved steps of a problem

\section{ACKNOWLEDGEMENT}

I want to acknowledge all authors of the journal articles that was used to complete this research work, also the assistance gotten from the computer laboratory of Adamawa State University, Mubi

\section{REFERENCES}

[1] Sherman, J. H, Richardson, I. L., \& Yard J. G. (2014). Why do students struggle with Mathematics, Pearson Allyn Bacon Prentice Hall.

[2] Mohan, R. (2013), Network Analysis and Remote Application Control Software based on Client-Server Architecture, International Journal of Computer Applications, 68 (12), 0975 - 8887.

[3] Caprotti, O., Cohen A. M., Cuypers H. and Sterk H. S (2002). OpenMath Technology for Interactive Mathematical Documents. ResearchGate.

[4] Josiah, A., \& Obi, N. (2012). Framework for Deploying Client/Server Distributed Database System for effective Human Resource Information Management Systems in Imo State Civil Service of Nigeria. International Journal of Academic Research in Business and Social Sciences $2(8)$.

[5] Florence, M. L., \& Swamydoss, D. (2011) Security Issues in Computer Network Architecture. Journal of Global Research in Computer Science, 2 (7).

[6] Arkorful, V., \& Abaidoo, N. (2014), The role of elearning, the advantages and disadvantages of its adoption in Higher Education. International Journal of Education and Research, 2 (12)

[7] Swan, M. (2005). Improving learning in mathematics: challenges and strategies, University of Nottingham.

[8] Jensen, F. M. (2014) Formal Verification of Distributed Programs using Session Types and Coq.

[9] Nguyen, Q., A. (2011). Development of A Framework for Structural Optimization Using Parallel Computers, Chair of Computing in Engineering, Faculty of Civil and Environmental Engineering, Ruhr University of Bochum

[10] Brandt, A., Thomas B., and Yorgov, D. (2015). Accessing the CU Denver Math Server. 